\title{
Outcome of newborns born to mothers with prolonged rupture of membrane
}

\author{
Thayi $\mathbf{S}^{1}$, Paramahamsa $\mathbf{R}^{2}$ \\ ${ }^{1}$ Dr. Sowmya Thayi, Post Graduate in Paediatrics, ${ }^{2}$ Dr. Ramakrishna Paramahamsa, Professor of Paediatrics, both \\ authors are affiliated with GSL Medical College and General Hospital, Rajahmundry, Andhra Pradesh, India.
}

Address for Correspondence: Dr. Sowmya Thayi, Email: drtsowmya.md@gmail.com

\begin{abstract}
Introduction: Prolonged rupture of membrane (PROM) is an important risk factor for early onset neonatal sepsis (EONS), which is associated with increased neonatal morbidity and mortality. This study is mainly done to know the outcome of neonates born to mothers with history of PROM. Methods: This study was conducted from January 2015 to December 2015 for a period of 12 months, in GSL medical college and hospital, Rajahmundry. Selection of cases-All the neonates born to mothers with history of prolonged rupture of membranes $>18 \mathrm{hrs}$ were included in our study. Required investigations were done and followed during their hospital stay. Results: Out of 200 neonates born to mothers with PROM, 60 had clinical sepsis, 29 had confirmed sepsis. Out of 29 confirmed cases of sepsis, positive blood culture was seen in 22 cases. The incidence of EONS in present study was found to be $14.5 \%$. In present study neonatal sepsis had a higher rate of incidence in preterm neonates (61.5\%) than in term neonates (38.5\%). The incidence of neonatal sepsis was found to be lower in neonates born to mothers who took prenatal antibiotic treatment $(9.25 \%)$ as compared to neonates born to mothers who did not receive prenatal antibiotic treatment $(20.65 \%)$. Respiratory distress was the commonest presenting clinical sign (33 cases). Out of all the five Sepsis screen parameters C-Reactive Protein has the highest sensitivity (90.90\%). Conclusion: Preventive measures should focus on recognition of these high-risk infants with prompt laboratory screening for sepsis and early institution of empirical antibiotic based on local data
\end{abstract}

Keywords: Prolonged rupture of membranes, Early onset neonatal sepsis, Sepsis screen, Blood culture

\section{Introduction}

Prolonged rupture of membrane (PROM) is an important risk factor for early Onset neonatal sepsis (EONS), which is associated with increased neonatal morbidity and mortality. This study is mainly done to know the outcome of neonates born to mothers with history of PROM.

According to the World Health Organization, approximately four million neonates die annually [1] with a global neonatal mortality rate of 23/1,000 live births [2]. About a million of these deaths are attributable to neonatal infection [3]. The incidence of neonatal sepsis according to the data from National Neonatal Perinatal Data base (NNPD 2002-03) is 30 per 1000 live births. It was reported as high as three times this number in developing countries compared to developed countries [4]. Prolonged rupture of

Manuscript received: $4^{\text {th }}$ October 2016

Reviewed: $15^{\text {th }}$ October 2016

Author Corrected; $25^{\text {th }}$ October 2016

Accepted for Publication: $11^{\text {th }}$ November 2016 membrane (PROM), defined as rupture of membrane lasting more than 18 hours before labor, is found in approximately $8 \%-10 \%$ of all pregnancies $[5,6]$. PROM is an important risk factor for both early onset neonatal sepsis (EONS) [7,8] and preterm births [9].

PROM is significant not only in perinatal morbidity and mortality, but also in the long term neonatal complications and sequelae in survived neonates [10].

Improved prenatal care and antenatal antimicrobial treatment of women with a history of PROM have significantly improved neonatal outcome in association with early detection of sepsis and its aggressive management in neonates $[10,11]$.

The evaluation of neonatal sepsis is important and there is an urgent need to know whether the baby has sepsis so as to institute treatment as quickly as possible. 


\section{Material and Methods}

This study was conducted from January 2015 to December 2015 for a period of 12 months, in GSL medical college and hospital, Rajahmundry.

Selection of cases- All the neonates born to mothers with history of prolonged rupture of membranes $>18 \mathrm{hrs}$ were included in our study.

A detailed history was taken including age, parity, Obstetric history of the mother with emphasis on exact time of rupture of membranes, duration history and antibiotics before labour were evaluated. Detailed birth history including resuscitation details, Apgar score and gestational age assessment were evaluated.

In examination of the neonate the pulse, respiratory rate, CFT and temperature were noted followed by systemic examination. Required investigations are done and followed during their hospital stay.

Inclusion criteria: All neonates born to mothers with Prolonged rupture of membranes more than 18 hours in GSL medical college and hospital, Rajahmundry.

\section{Exclusion criteria:}

1. Antepartum hemorrhage

2. Toxemia of pregnancy
3. Major medical illness to the mother other than infections.

4. Mothers with PROM $<18 \mathrm{hrs}$.

5. Neonates with major congenital malformations

6. Neonates presenting with symptoms after $72 \mathrm{hrs}$ after birth

7. Neonates who underwent any mode of resuscitation other than routine care.

Following investigations were carried out :

1. Hb\% was estimated by automated analyzer

\section{Sepsis screen panel}

- Total leukocyte count (TLC) estimated by automated analyser.

- Differential leucoyte count (DLC) done by peripheral smear

- Band count estimated by peripheral smear

- Micro ESR

- CRP semi quantitive estimation by latex agglutination technique

\section{Blood culture and sensitivity}

Diagnostic criteria- Diagnosis of neonatal sepsis is made in neonates having clinical signs and symptoms consistent with sepsis having any 2 parameters of sepsis screen positive or neonate with positive blood culture.

\section{Results}

A total of 200 neonates born to mothers with history of PROM are included in the present study.

Out of 200 neonates, 60 neonates had clinical signs and symptoms consistent with sepsis and are included in the category "clinical sepsis"

Out of 200 neonates 29 neonates had confirmed sepsis ie clinical features + either sepsis screen and/or blood culture positive and are included in the category "confirmed sepsis"

In the present study it was observed male neonates with confirmed sepsis were more $62 \%$ compared to female neonates $38 \%$. The incidence of sepsis is higher in low birth weight neonates (66\%) compared to normal birth weight babies (34\%) and neonatal sepsis had a higher rate of incidence in preterm neonates $(61.5 \%)$ than in term neonates $(38.5 \%)$. And was also found to be higher in neonates born by normal vaginal $(68.5 \%)$ delivery compared to caesarean section. ( $31.5 \%)$

In present study there is significant difference in the incidence of EONS in neonates born to mothers who took prenatal antibiotic (9.25\%) and those who did not receive prenatal antibiotic (20.65\%). From our study, importance of prenatal antibiotics preventing Early onset neonatal sepsis is well noted.

Incidence of sepsis was found to be higher in neonates born by normal vaginal $(68.5 \%)$ delivery compared to caesarean section $(31.5 \%)$. 


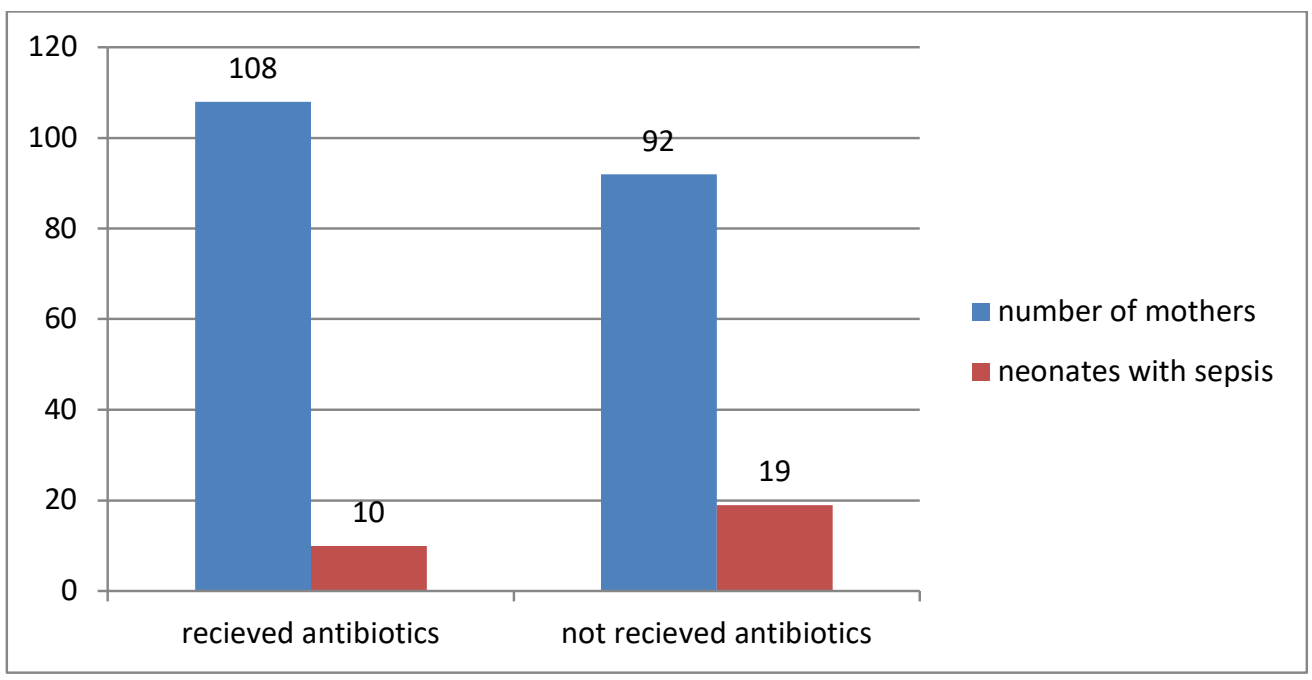

Distribution of cases in relation to prenatal antibiotic treatment

Out of 200 neonates born to mothers with PROM, 60 neonates had more than 3 symptoms/ signs consistent with sepsis. Hence number of neonates included in the category of clinical sepsis are- 60

Out of 60 cases with clinical signs and sepsis positive, respiratory distress was the commonest presenting clinical sign ( 33 cases) followed by poor feeding ( 29 cases), seizures were observed least in the present study with only 4 among 60 clinical sepsis cases.

Table 1: Distribution of cases based on presenting clinical signs and symptoms.

\begin{tabular}{|c|c|c|c|}
\hline S. No & Presenting Clinical Sign / Symptom & Number & Percentage \\
\hline 1 & Respiratory Distress & 33 & $55 \%$ \\
\hline 2 & Poor Feeding & 29 & $48.33 \%$ \\
\hline 3 & Lethargy & 26 & $46.33 \%$ \\
\hline 4 & Jaundice & 22 & $36.66 \%$ \\
\hline 5 & Fever & 19 & $31.66 \%$ \\
\hline 6 & Excessive Cry & 15 & $25 \%$ \\
\hline 7 & Absent Moro Reflex & 12 & $20 \%$ \\
\hline 8 & Hypothermia & 10 & $16.66 \%$ \\
\hline 9 & Seizures & 4 & $6.66 \%$ \\
\hline
\end{tabular}

- Prolonged duration of PROM more than $18 \mathrm{hrs}$ is an indication for performing sepsis screening. Hence sepsis screen was done on all the neonates of PROM mothers included in our study group.

- Total number of neonates who underwent sepsis screen- 200

- Sepsis Screen is considered positive if any neonate shows any 2 of the 5 parameters positive.

- Total number of neonates with positive sepsis screening-29

- All the 29 neonates also had clinical signs and symptoms consistent with neonatal sepsis

- Out of all the five sepsis screen parameters CRP has the highest sensitivity (90.90\%), with 26 cases showing positive CRP in 29 cases of confirmed sepsis. Followed by Micro ESR (65.51\%) with 19 cases positive in 29 cases of confirmed sepsis.

- Blood collected from all the neonates showing symptoms and signs consistent with clinical sepsis..

- Out of 60 neonates showing clinical signs and symptoms. Blood culture was positive only in 22 cases.

Out of 26 cases of CRP positive, nearly 20 cases had blood culture positive thus indicating, CRP correlates well with blood culture with highest sensitivity among all the other sepsis screen parameters. 
Table 2: Sepsis screen parameters and their statistical data.

\begin{tabular}{|c|c|c|c|c|}
\hline Parameters & Sensitivity & Specificity & $\begin{array}{c}\text { Positive predictive } \\
\text { value }\end{array}$ & $\begin{array}{c}\text { Negative predictive } \\
\text { value }\end{array}$ \\
\hline Total WBC count & $59.09 \%$ & $71.42 \%$ & $86.66 \%$ & $50.0 \%$ \\
\hline $\begin{array}{c}\text { Absolute neutrophil } \\
\text { count }\end{array}$ & $63.63 \%$ & $72.34 \%$ & $85.30 \%$ & $33.33 \%$ \\
\hline Band count & $59.09 \%$ & $57.14 \%$ & $81.25 \%$ & $30.76 \%$ \\
\hline Micro ESR & $72.72 \%$ & $56.03 \%$ & $84.21 \%$ & $31.62 \%$ \\
\hline CRP & $90.90 \%$ & $85.71 \%$ & $95.23 \%$ & $75.0 \%$ \\
\hline
\end{tabular}

Out of 22 cases of blood culture positive, staphylococcus aureus was isolated in 10 cases and has the highest percentage followed by coagulase negative staphylococcus aureus (CONS)

Table 3: Bacterial pathogens isolated in blood culture of neonates.

\begin{tabular}{|c|c|c|c|}
\hline S. No & Bacterial Isolate & No. of Cases & Percentage \\
\hline 1. & Staphylococcus aureus & 10 & $45.45 \%$ \\
\hline 2. & Coagulase negative staph aureus & 6 & $27.27 \%$ \\
\hline 3. & Klebsiella & 3 & $13.6 \%$ \\
\hline 4. & E.coli & 3 & $13.6 \%$ \\
\hline & Total & $\mathbf{2 2}$ & $\mathbf{1 0 0 \%}$ \\
\hline
\end{tabular}

\section{Discussion}

In present study male babies have more incidence of early onset neonatal sepsis compared to female babies which is consistent with Hitesh J Assudani study [12].

Aletayeb S et al [13] and Celiciac C et al [14] study also observed incidence of sepsis is more in male babies compared to female babies.

In Betty Chacko and Inderpreet Sohi [15] study incidence of neonatal sepsis was similar to male and female neonates which is not consistent with the present study. There was significant difference in the incidence of sepsis between male neonates $(62 \%)$ and female neonates $(38 \%)$. ( $p$ value $<0.05)$. Bias for male sex, place of study, sample including other factors may be responsible for increased number of male cases in these studies. We observed that the incidence of neonatal sepsis was more in low birth weight neonates $<2500 \mathrm{gm}$ (66.1\%) compared to normal birth weight neonates (33.9 5). (p value $<0.05)$.

Similar results were obtained in other studies such as Yasmeen J Al Bayaa et al study [16] (76.3\% in LBW neonates vs $23.7 \%$ in normal birth weight neonates) and Rajashri Basuet al study [17] (74.87\% vs 23.7\%). However in Woranart et al study [18] incidence of neonatal sepsis was almost similar in both low birth weight and normal birth weight neonates. In present study there was significant difference in preterm neonates $(61.5 \%)$ compared to term babies (38.5\%). (p value $<0.05$ ). Other authors [19] had similar results as the present study. Due to immature immune function, premature babies are more prone to infections compared to term

In present study there was significant difference in the incidence of neonatal sepsis in neonates born by normal vaginal delivery $(68.5 \%)$ vs caesarean section $(31.5 \%)$ ( $\mathrm{p}$ value $<0.05$ ). Other studies which are consistent with the present study are Kifah Al-Q Fatin-Al awayshah study [19] in which incidence of neonatal sepsis in neonates born by Normal vaginal delivery are (54\%) compared to neonates born by caesarean section (20\%).

Sanyal Mukherjee [20] and Kodkany at al also observed [21] much greater difference in the incidence of neonatal sepsis in neoantes born by normal vaginal delivery compared to caesarean section.

In present study Respiratory distress (54.5\%) was the commonest presenting symptom followed by refusal of feeds (47.5\%) and lethargy. (43.5\%)

In Jain N K et al [23] also observed respiratory distress $(42.6 \%)$ as the commonest presenting symptom. However in omer et al observed [22] fever (63.33\%) as commonest presenting sign followed by respiratory distress. 
Earliest clinical features of neonatal sepsis are often subtle and non specific therefore a high index of suspicion is needed for early diagnosis specially so if risk factors are also present Clinical features and further course in neonatal sepsis depends on various factors like birth weight, place of delivery, age of newborn, intervention in preventable factors for sepsis, availability, accessibility, affordability and timely referral of baby to an appropriate centre. Therefore variation in different parameters may be observed in various studies.

We observed that the percentage of neonatal sepsis is higher in mothers with PROM who did not receive prenatal antibiotics $(20.65 \%)$ compared to mothers who had prenatal antibiotic intake $(9.25 \%)$.

Similar results were obtained by Kifah Al-Q Fatin -Al awayshah study [19]. The percentage of neonatal sepsis is only $4.4 \%$ in neonates born to mothers who received prenatal antibiotics compared to $11 \%$ neonates whose mothers did not receive prenatal antibiotics for PROM. There are several studies in which women presenting with preterm PROM without labor were randomized to receive an antibiotic or placebo. Over all, these studies have shown that antibiotics prolonged the latency period until delivery, reduced maternal choriomnionitis and reduced neonatal complications, including respiratory distress syndrome, IVH, pneumonia and sepsis as in present study newborns whose mothers received antibiotic before have good outcome.

Antibiotic have become an important part of the recent advances in the treatment of PROM. Many studies have demonstrated that antibiotic therapy prolong pregnancy latency time and decreases maternal and neonatal morbidity

The sensitivity and specificity of CRP in present study is higher with $90.90 \%$ and $85.71 \%$ respectively. The present study is consistent with chauhan et al [24] with a sensitivity and specificity of $92.30 \%$ and $85.71 \%$ respectively.

But in Boma A West et al study [25] sensitivity and specificity are lower with $74 \%$ which is not consistent with the present study. CRP passes the placenta only in very low quantities; therefore, any elevation in the neonate always represents endogenous synthesis. De novo hepatic synthesis starts very rapidly after a single stimulus with serum concentrations rising above $5 \mathrm{mg} / 1$ by about 6 hours and peaking around 48 hours.
In diagnosis of early onset sepsis previous studies reported on widely differing sensitivities and specificities of CRP ranging from $29 \%$ to $100 \%$ and from $6 \%$ to $100 \%$, respectively. These extreme variations are a result of different reference-values, test methodologies, patient characteristics and inclusion criteria, number of samples taken, and sampling time. Furthermore, definitions of sepsis widely differ between studies making serious comparisons hardly possible.

On the other hand a raised CRP is not necessarily diagnostic for sepsis, as elevations may as well occur due to the physiologic rise after birth or non infection associated conditions. Therefore, concerns were raised about the reliability of CRP during the early stage of the disease being neither able to diagnose nor to rule out an infection with certainty.

Compared to other studies, blood culture positivity is slightly lower with $36.66 \%$ in the present study. In kayange et al study [26] positive blood culture was seen in 57 among 121 clinical sepsis cases, and in Eman M. Rabie et al study [27], blood culture positivity was seen in nearly $40.7 \%$ clinical sepsis cases.

Low blood culture positivity in the present study may be due to small sample size compared to other two studies. This is similar to other studies done in Bangaladesh and Uganda [28,29]. Incidence of Early onset Neonatal Sepsis (EONS) in present study is $14.5 \%$ which is slightly lower compared to other studies. Nili and AA Shams Ansari study [30] shows $18.5 \%$ which is slightly above the present study. Ratanakorn W et al study [31] shows the highest incidence about $27.9 \%$.

These differences are probably due to use of different criteria for diagnosis of neonatal sepsis and/or inclusion of coagulase negative staphylococcus and other contaminants.

In present study Staphylococcus aureus was the commonest isolate $(45.45 \%)$ followed by Coagulase negative staphylococcal aureus (CONS) (27.27\%).

This was contrary to NNPD-2002-2003, where klebsiella was the commonest isolate followed by staph aureus. In another study conducted by Shashikala. S et al [32] on 242 babies klebsiella was the commonest isolate.

Funding: Nil, Conflict of interest: Nil Permission from IRB: Yes 


\section{References}

1. Högberg U. The World Health Report 2005: "make every mother and child count" - including Africans. Scand J Public Health. 2005;33(6):409-11.

2. World Health Report (2011): Level and trends in child mortality. Geneva: WHO 2011.

3.Black RE, Cousens S, Johnson HL, Lawn JE, Rudan I, Bassani DG, Jha P, Campbell H, Walker CF, Cibulskis R, Eisele T, Mathers C; Child Health Epidemiology Reference Group of WHO and UNICEF. 2010 Jun 5;375(9730):1969-87. doi: 10.1016/S01406736(10)60549-1. Epub 2010 May 11

4. Mahmood A, Fazal ur Rehman, Chughtai F. A survey of infection control practices in the delivery room and nursery to investigate and control the high rate of neonatal sepsis: an experience at a secondary care hospital. J Pak Med Assoc. 2008 May;58(5):237-40.

5. Popowski T, Goffinet F, Maillard F, Schmitz T, Leroy S, Kayem G. Maternal markers for detecting early-onset neonatal infection and chorioamnionitis in cases of premature rupture of membranes at or after 34 weeks of gestation: a two-center prospective study. BMC Pregnancy Childbirth. 2011 Apr 7;11:26. doi: 10.1186/1471-2393-11-26.

6. Kilbride HW, Thibeault DW. Neonatal complications of preterm premature rupture of membranes. Pathophysiology and management. Clin Perinatol. 2001 Dec;28(4):761-85.

7. Mercer BM, Goldenberg RL, Das AF, Thurnau GR, Bendon RW, Miodovnik M, Ramsey RD, Rabello YA; National Institute of Child Health and Human Development Maternal-Fetal Medicine Units Network. What we have learned regarding antibiotic therapy for the reduction of infant morbidity after preterm premature rupture of the membranes. Semin Perinatol. 2003 Jun; 27(3):217-30. Review.

8. Medina TM, Hill DA. Preterm Premature Rupture of Membranes: Diagnosis and management. Am Fam Physician 2006;73:659-664.

9. Boskabadi H, Maamouri G, Mafinejad S (2011) Neonatal Complications Related with Prolonged Rupture of Membranes Macedonian Journal of Medical Sciences. 2011 Mar 15; 4(1):93-98. doi:10.3889/MJMS. 1857-5773.2011.0159
10. Mercer B. Antibiotics in the management of PROM and preterm labor. Obstet Gynecol Clin North Am. 2012 Mar;39(1):65-76. doi: 10.1016/j.ogc.2011.12.007. Epub 2012 Jan 28.

11. Al-Qa K, Al-Awaysheh F. Neonatal outcome and prenatal antibiotic treatment in premature rupture of membranes. Pak J Med Sci. 2005; 21(4): $441-444$

12. Assudani HJ, Gusani JK, Mehta SJ, Agravat HH, Kothari K. Bacteriological profiles of septicaemia in neonates at tertiary care hospital, Gujarat, India. J Res Med Den Sci. (2015), [cited December 23, 2016]; 3(2): 148-151. doi:10.5455/jrmds.20153212

13. Aletayeb SM, Khosravi AD, Dehdashtian M, Kompani F,Mortazavi SM, Aramesh RM. Identification of bacterial agents and antimicrobial susceptibility of neonatal sepsis: A 54-month study in a tertiary hospital. African Journal of Microbiology Research 2011;5(5): 528-31.

14. Cecilia CM, Mary AC, Elizabeth EG, Jonathan GL, Joanne JL et al. Etiology of neonatal sepsis in five urban hospitals in the Philippines. PIDSP Journal 2011;12(2):75-85

15. Early Onset Neonatal Sepsis Betty Chacko and Inderpreet Sohi Neonatology Unit, Department of Pediatrics, Christian Medical College \& Hospital, Ludhiana, Punjab, India. Indian J Pediatr 2005;72(1): 23-26.

16. Yasmeen J. Al-Bayaa, Nedhal S.Ayoub, Sevan Najem Alwan. Relationship between Neonatal septicemia and birth weight. Fac Med Baghdad 2012; 54(2): 151-153

17. Rajarshi Basu, Syamalkumar Bandyopadhyay. Study on Correlation between Sepsis Screening Blood Culture in Neonatal Sepsis. IOSR Journal of Dental and Medical Sciences May 2014; 13(5): $52-56$.

18. Woranart Ratana Korn M.D., Witsanuchai Sri Jariya RPH. Ph.D (C) et al Incidence of neonatal infection in newborn infants with matneral history of premature rupture of membranes (PROM) for 18 houirs or longer. J Med Assoc. Thai 2005; 8 (7): 973-978. 
19. Kifah Al-Qa Qa and Fatin Al-Awaysheh. Neonatal outcome and prenatal antibiotic treatment in premature rupture of membranes. Pak J Med Sci. 2005;21: 441-444

20. Sanyal MK and Mukherjee TN. premature rupture of membrane; an assessment from a rural medical college of West Bengal; J. Obstet Gynecol India 1990; 40(4): $623-628$

21. Kodkany BS, Telang MA. Premature rupture of membranes. A study of 100 cases. J. Obstet Gynecol India 1991; 41(4): 492-496

22. Sudan. Omer Saeed Magzoub, Mohamed Awadelkrim Ahmed, Yahia Shakir Abdelgadir. Clinical presentation of neonatal sepsis in paediatric ward at Khartoum North Teaching Hospital.Basic Research Journal of Medicine and Clinical Sciences.April 2015;4 (4);116-120.

23. Jain NK, Jain VM, Maheshwari S. Clinical profile of neonatal sepsis. Kathmandu Univ Med J (KUMJ). 2003 Apr-Jun;1(2):117-20.

24.Setal B Chauhan, Viren Vaghasia, Bimal B Chauhan.C-Reactive Protein (CRP) in Early Diagnosis of Neonatal Septicemia. Natl J Med Res. 2012; 2(3): 276-278.

25. Boma A West, Oliemen Peterside, Rosemary O Ugwu,and Augusta U Eneh. Prospective evaluation of the usefulness of C-reactive protein in the diagnosis of neonatal sepsis in a sub-Saharan African region. Antimicrob Resist Infect Control. 2012;1;22-26.

26. Neema Kayange, Erasmus Kamugish, Damas L Mwizamholya1, Seni Jeremiah and Stephen E Mshana. Kayange et al. Research article Predictors of positive blood culture and deaths among neonates with suspected neonatal sepsis in a tertiary hospital, Mwanza- Tanzania. BMC Pediatrics 2010; 10:39

27. Eman M. Rabie Shehab El-Din, Mohamed M. Adel El-Sokkary, Mohamed Reda Bassiouny, and Ramdan Hassan. Epidemiology of Neonatal Sepsis and Implicated Pathogens: A Study from Egypt Hassan. BioMed Research International. 2015;20:1-11

28. A. S. Ahmed, M. A. Chowdhury, M. Hoque, and G. L. Darmstadt. Clinical and bacteriological profile of neonatal septicemia in a tertiary level pediatric hospital in Bangladesh. Indian Pediatrics 2002;39(11):1034-39.

29. J. Mugalu, M. K. Nakakeeto, S. Kiguli, and D. H. KadduMulindwa. Aetiology, risk factors and immediate outcome of bacteriologically confirmed neonatal septicaemia in Mulago hospital, Uganda. African Health Sciences. 2006; 6(2):120- 126.

30. F-Nilli and Shams Ansari A. Neonatal Complications of premature rupture of membrane. Acta Medica Iranica 2003;41(3) 176.

31. Ratanakorn W, Srijariya W, Chamnanvanakij S, Saengaroon P. Incidence of neonatal infection in newborn infants with a maternal history of premature rupture of membranes (PROM) for 18 hours or longer by using Phramongkutklao Hospital Clinical Practice Guideline (CPG). J Med Assoc Thai 2005 Jul;88 (7): 973-8.

32.Shashikala S. Tallur A. V. Kasturi,Shobha D.Nadgir, B. V. S. Krishna. Clinico-bacteriological study of neonatal septicemia in Hubli. Original Article. The Indian Journal of Pediatrics. 2000; 67(3); 169-174.

\section{How to cite this article?}

Thayi S, Paramahamsa R. Outcome of newborns born to mothers with prolonged rupture of membrane.Int.J Pediatr Res.2016;3(11):835-841.doi:10.17511/ijpr.2016.i11.12. 